\title{
Liquid-junction Free Reference Electrode Integration into Amperometric Sensor Probe Using Closed Bipolar Electrode System
}

\author{
Kumi Y. Inoue ${ }^{1}$, Ryoto Tsuga ${ }^{2}$, Siti Masturah Binti Fakhrunddin ${ }^{1}$, Tomokazu Matsue ${ }^{1}$ \\ ${ }^{1}$ Graduate School of Environmental Studies, Tohoku University, 6-6-11-604 Aramaki, Aoba, Sendai \\ 980-8579, Japan, \\ ${ }^{2}$ School of Engineering, Tohoku University, 6-6-11-604 Aramaki, Aoba, Sendai 980-8579, Japan
}

\begin{abstract}
:
A reference electrode used for electrochemical measurement generally has a liquid junction to allow the ion transport between an internal electrolyte and a test liquid for electrical conduction between the reference electrode and the test liquid. However, the liquid junction often causes troubles such as clogging and test liquid contamination. Previously, we proposed a galvanic cell system without liquid junction using a close bipolar electrode system. In this research, we fabricated a probe type amperometric sensor integrated with liquid-junction free reference electrode system based on the knowledge obtained by the previous study. This compact device successfully demonstrated to be used for ferrocenemethanol quantification in the range 0.1-0.5 mM.
\end{abstract}

Key words: bipolar electrode system, reference electrode, amperometry, probe, liquid junction

\section{Introduction}

Liquid junction is required for reference electrode system in order to complete the electrical circuit for the potential measurement by passing thorough the ions between the reference electrolyte and sample. Any types of liquid junction including salt bridge, ceramic plug and ground sleeve have problems of sample contamination and stuck of junction. Furthermore, it is very hard to fabricate a liquid junction on an integrated system by microfabrication process. Previously we proposed a galvanic cell system without liquid junction for accumulation step of substitutional stripping voltammetry using a closed bipolar electrode system (cBPES)[1]. The cBPES is a system composed of a bipolar electrode connecting between two separated electrochemical cells containing a driving electrode in each cell. By applying an adequate potential between the two driving electrodes, two conjugate electrochemical reactions are occurred at the each end of the bipolar electrode. Under the condition that the target redox reaction at an end of the bipolar electrode proceeds as rate limiting process of the whole system, cBPES can be used as a quantitative measurement system. In this paper, we report an amperometric sensor probe with integrated liquid-junction free reference electrode by applying the same system.

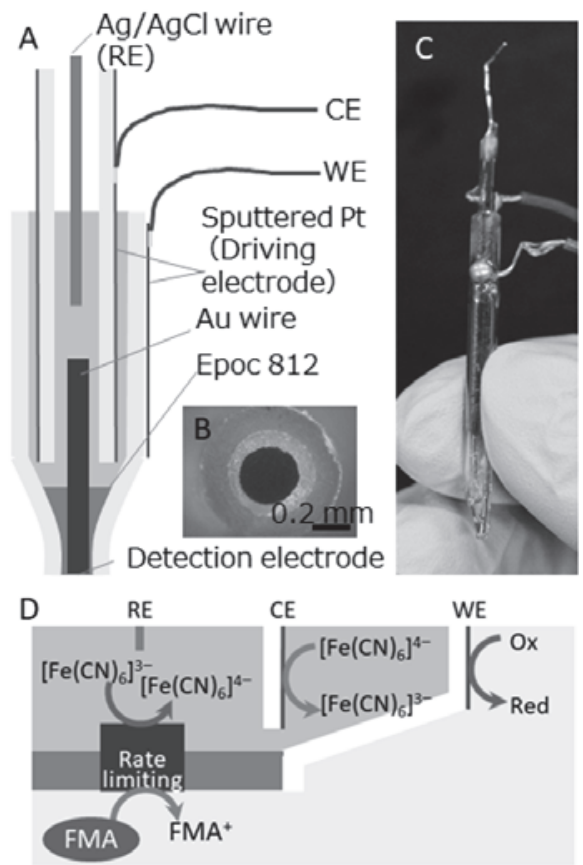

Fig. 1. Schematic illustration of fabricated probe (A), microscope image of detection electrode (B), photograph of a fabricated probe (C) and schematic illustration of electrochemical reaction on FMA sensing. 


\section{Experimental}

First we fabricated a probe device shown Fig. $1 \mathrm{~A}-\mathrm{C}$. A gold wire with a diameter of $200 \mu \mathrm{m}$ cut to $2 \mathrm{~cm}$ was inserted into a glass tube $(O D=3$ $\mathrm{mm}, I D=1.8 \mathrm{~mm}$ ) whose tip was thinned with heat. The tip end was filled with an embedding resin for electron microscope (Epoch 812) to fix the gold wire and to inhibit mass transfer between inside and outside of the glass tube. The outside surface of the glass tube was sputteredon the half plane with the tip part left, to use as a driving electrode connected to the WE terminal of a potentiostat. After dicing the tip, the tip surface was polished with alumina powder to obtain a gold disk electrode used as a sensor electrode. Inside the glass tube, a thinner glass tube $(O D=1.65 \mathrm{~mm}, I D=1.1 \mathrm{~mm})$ with $\mathrm{Pt}$ sputtered on the entire outer surface was inserted to use as a driving electrode connected to the CE terminal of a potentiostat. An $\mathrm{Ag} / \mathrm{AgCl}$ wire with a diameter of $300 \mu \mathrm{m}$ was inserted into a thinner glass tube and connected to the RE terminal of a potentiostat to use as a reference electrode. A mixture solution of 2.5 $\mathrm{mM} \mathrm{K}_{4}\left[\mathrm{Fe}(\mathrm{CN})_{6}\right], 2.5 \mathrm{mM} \mathrm{K}_{3}\left[\mathrm{Fe}(\mathrm{CN})_{6}\right]$ and saturated $\mathrm{KCl}$ was added into the tube as an internal solution.

Next, cyclic voltammetry measurements of ferrocene methanol (FMA) were carried out using the prototype device. 0.1-0.5 mM FMA aqueous solution (containing $0.1 \mathrm{M} \mathrm{KCl}$ ) was used as analyte solutions. When the FMA oxidation progresses, the reaction shown in Fig. 1D occurs at each electrode. Therefore, the peak caused by the FMA oxidation appears as a negative current when sweeping the potential of the WE terminal in the negative potential direction unlike the usual case. In consideration of this, the potential of the WE terminal was swept in the direction of $0.3 \rightarrow 0 \rightarrow 0.4 \rightarrow 0.3 \mathrm{~V}$ at a scan rate of $20 \mathrm{mV} / \mathrm{s}$. Then, the amperometry was carried out using the prototype device by applying $0.05 \mathrm{~V}$ to the WE terminal vs. RE. The average of the amperometric currents at 22-25 s were used for calibration plot.

\section{Results and Discussion}

The obtained voltammograms are shown in Fig. $2 \mathrm{~A}$. The peaks around $0.18 \mathrm{~V}$ were increased with increasing the concentration of FMA in the range of 0.1-0.5 mM. This result shows that the prototype device successfully worked as our concept. Fig. 2B shows the amperograms for 0.1-0.5 mM FMA. The amperometric currents were also increased with increasing the concentration of FMA in the range of 0.1-0.5 mM. We successfully obtained linear calibration plot for FMA by employing the average of the amperometric currents at 22-25 s (Fig. 1C).
A

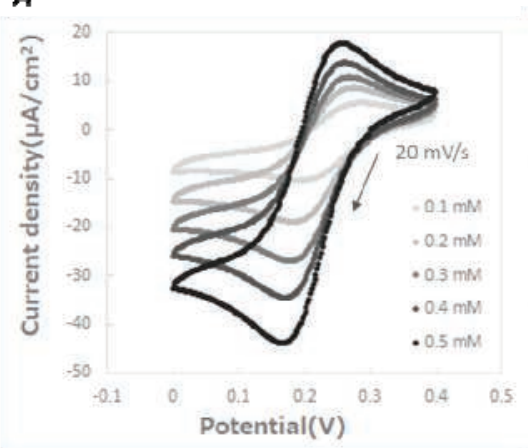

B

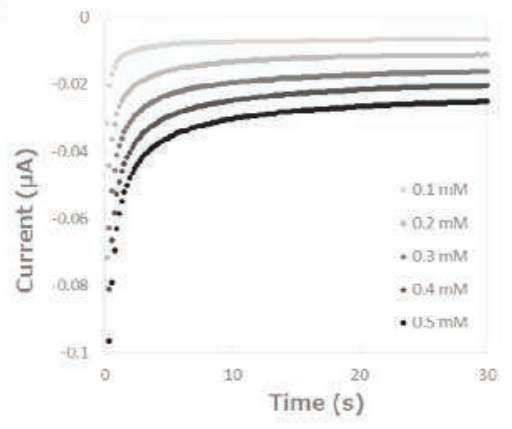

C

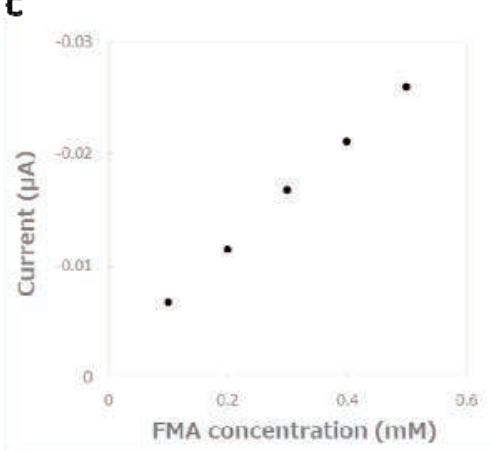

Fig.2. Cyclic voltammograms $(A)$ and amperograms $(B)$ for 0.1-0.5 mM ferrocenemethanol. The calibration curve (C) was prepared by adopting the average of currents at 22-25 s in amperometry.

\section{Conclusion}

In this study, we developed an amperometric sensor probe with integrated liquid-junction free reference electrode by applying a close type bipolar electrode system. We successfully detected 0.1-0.5 mM FMA as a model for quantitative analysis in prototype devices. This device can be used for measurement in a dirty environment or in a system where chloride ion contamination is a problem, taking advantage of the liquid-junction free reference electrode inte- grated device. We will apply this sensor into a biosensor by enzyme modification, in order to use as a urine sensor for daily healthcare

\section{References}

[1] S. Takano, K. Y. Inoue, M. Ikegawa, Y. Takahashi, K. Ino, H. Shiku, T. Matsue, Liquidjunction-free system for substitutional stripping voltammetry using a closed bipolar electrode system, Electrochem. Commun., 66, 34-37 (2016); DOI: 10.1016/j.elecom.2016.02.014 